\title{
Interação entre características de argilas e parâmetros de processamento sobre propriedades tecnológicas de corpos cerâmicos
}

\section{(Interaction between clays characteristics and processing parameters on technological properties of ceramic bodies)}

\author{
R. C. dos Santos, T. R. da Silva, G. de A. Neves, R. S. de Macedo, R. R. Menezes, L. N. de L. Santana \\ Programa de Pós-Graduação em Ciência e Engenharia de Materiais, Universidade Federal de Campina \\ Grande, Av. Aprígio Veloso 882, Campina Grande, PB 58109-970 \\ renato.materiais@gmail.com,tamiris.rodrigues1@gmail.com,gelmires.neves@ufcg.edu.br, \\ reginaldo.severo@ufcg.edu.br,romualdo.menezes@ufcg.edu.br,lisiane.navarro@ufcg.edu.br
}

\begin{abstract}
Resumo
As propriedades dos produtos cerâmicos estão intimamente relacionadas às características iniciais das matérias-primas, mas também aos parâmetros e condições de processamento. Dentro desse contexto, este trabalho teve como objetivo avaliar a influência das características de argilas e sua interação com as variáveis de processamento sobre as propriedades tecnológicas de corpos de prova. Foram utilizadas argilas provenientes dos estados de Pernambuco e da Paraíba. As argilas foram beneficiadas e em seguida submetidas a ensaios de caracterização. Os corpos de prova foram conformados por prensagem e por extrusão, secos a $110^{\circ} \mathrm{C}$ e em seguida submetidos à queima nas temperaturas de $800,900,1000,1100 \mathrm{e} 1200^{\circ} \mathrm{C} \mathrm{com}$ taxa de aquecimento de $5^{\circ} \mathrm{C} / \mathrm{min}$ e tempo de queima de 60 e $180 \mathrm{~min}$. Em seguida foram avaliadas as propriedades tecnológicas (retração linear de queima, absorção de água e resistência à flexão em três pontos) e as fases formadas para os diferentes tratamentos térmicos. Pôde-se concluir que a interação das características granulométricas, mineralógicas e químicas, assim como os parâmetros de processamento, principalmente o método de conformação, são determinantes para as propriedades tecnológicas da peça cerâmica.
\end{abstract}

Palavras-chave: argilas, parâmetros de processamento, propriedades tecnológicas.

\begin{abstract}
Properties of ceramics materials are closely related to the initial characteristics of the raw materials and also to the conditions and parameters of the processing. In this context, this study aimed to evaluate the influence of clays characteristics and their interaction with the processing variables on technological properties of fired specimens. Clays from the states of Pernambuco and Paraiba were used in this work. Clays were processed and then subjected to characterization tests. The specimens were produced by pressing and extrusion, dried at $110^{\circ} \mathrm{C}$ and then subjected to firing at temperatures of $800,900,1000,1100$ and $1200{ }^{\circ} \mathrm{C}$, with heating rate of 5 ${ }^{\circ} \mathrm{C} / \mathrm{min}$ and firing time of 60 and $180 \mathrm{~min}$. Then, the technological properties (linear firing shrinkage, water absorption, and threepoint flexural strength) and the phases produced under the different thermal treatments were analyzed. It was concluded that the interaction of particle size, mineralogical and chemical characteristics, as well as the processing parameters, especially the forming method, were determinants to the technological properties of ceramic bodies.
\end{abstract}

Keywords: clays, processing parameters, technological properties.

\section{INTRODUÇÃO}

O conhecimento das características das matériasprimas argilosas e de sua relação com os parâmetros de processamento na qualidade dos produtos cerâmicos vêm sendo muito estudados recentemente [1-6]. Esses estudos vêm sendo impulsionados pela necessidade de aprimoramento dos processos de fabricação, em virtude do uso de matérias-primas de menor qualidade atualmente ou por causa de demandas do mercado para otimização de desempenho ou de aplicação de peças cerâmicas produzidas com matérias-primas tradicionais.

$\mathrm{Na}$ cerâmica tradicional, conhecer as argilas e seus constituintes é de fundamental importância para o processo de fabricação, pois a presença e a quantidade de cada um dos seus componentes e o seu comportamento frente aos parâmetros de processamento possibilitam compreender e definir as propriedades do produto final $[7,8]$. Por outro lado, argilas de boa qualidade estão se exaurindo e o uso de materiais com baixas frações de argilominerais, principalmente caulinita, ou com elevadas frações de mica, ilita ou mesmo esmectita vem se tornando uma regra, juntamente como a necessidade de melhora do desempenho mecânico de blocos, telhas e revestimentos. Essa tendência exige que seja aprofundada a pesquisa na tecnologia cerâmica a fim de se ter os subsídios para produzir peças com 
melhor qualidade, usando matérias-primas aparentemente de menor qualidade. Nesse sentido, o processamento tem papel fundamental no sucesso deste desafio. O processo de fabricação de uma peça cerâmica é complexo e precisa ser analisado sempre de forma global, envolvendo todo o ciclo de processo. De forma geral, tem-se que o processamento é composto por uma sequência de etapas integradas, desde a escolha da matéria-prima até a queima da peça, sendo quatro as etapas principais: preparação da massa, conformação, secagem e queima. Todos esses estágios devem ser analisados e otimizados de forma conjunta, a fim de se obter a microestrutura necessária e desejada no produto final e, consequentemente, as propriedades mecânicas almejadas [9, $10]$.

Dentre esses estágios têm-se, particularmente, a conformação e a queima que podem, considerando as etapas de preparação da massa e de secagem já otimizadas, melhorar significativamente as propriedades do produto final se adequadamente executadas [11-13]. Para isso é necessário utilizar as rotas de processamento mais adequadas a cada matéria-prima e suas características físicas e mineralógicas, bem como aos requisitos técnicos e econômicos da peça final. Assim, pode-se variar a forma de conformação e as características do ciclo de queima a fim de se melhorar o desempenho de formulações produzidas com matériasprimas de menor qualidade. Dentro desse contexto, o objetivo deste trabalho foi avaliar a influência da interação das características químicas, mineralógicas e granulométricas de argilas, e o efeito de variáveis de processamento, processo de conformação e tratamento térmico, sobre propriedades tecnológicas de peças produzidas com matérias-primas argilosas.

\section{MATERIAIS E MÉTODOS}

Para o desenvolvimento deste trabalho foram utilizadas argilas oriundas de jazimentos da cidade de Caruaru (Bacia do Rio Ipojuca), estado de Pernambuco, denominadas amostras A e B, e da cidade de Juazeirinho (Bacia do Rio Taperoá), do estado da Paraíba, denominada amostra C. As argilas foram secas, desagregadas em triturador e moinho tipo martelo e em seguida submetidas a peneiramento em peneiras ABNT $\mathrm{N}^{\circ} 200$ (abertura de $0,074 \mathrm{~mm}$ ) e ABNT $\mathrm{N}^{\circ}$ 80 (abertura de $0,177 \mathrm{~mm}$ ). Após o beneficiamento foram caracterizadas através de: determinação de composição química, por fluorescência de raios $\mathrm{X}$ por energia dispersiva (Shimadzu, EDX-720); difração de raios X (Shimadzu, XRD
6000), com radiação $\mathrm{CuK \alpha}\left(40 \mathrm{kV}, 30 \mathrm{~mA}\right.$, passo de $0,02^{\circ}$, tempo de contagem de $0,6 \mathrm{~s}$ ); determinação da distribuição do tamanho de partículas (Cilas, 1064LD), utilizando-se hexametafosfato de sódio como agente dispersante e agitação por ultrassom; e determinação dos índices de plasticidade através do método de Casagrande, determinando-se o limite de liquidez (LL), o limite de plasticidade (LP) e o índice de plasticidade (IP) das argilas.

Foram confeccionados corpos de prova utilizando-se os processos de prensagem e extrusão. Para a conformação por prensagem foram adicionados $8 \%$ de água à massa e após 24 h, para homogeneização da umidade, foram confeccionados corpos de prova de $5,0 \mathrm{~cm} \times 1,5 \mathrm{~cm} \times 0,5 \mathrm{~cm}$, aplicando uma pressão uniaxial de $20 \mathrm{MPa}$ (Servitech, CT-335). Para a conformação dos corpos de prova por extrusão foram adicionados aproximadamente $25 \%$ de água e após 24 $\mathrm{h}$, para homogeneização da umidade, foram extrudados corpos de prova de $5,0 \mathrm{~cm} \times 1,5 \mathrm{~cm} \times 0,5 \mathrm{~cm}$ utilizando uma extrusora de laboratório (Verdés, 050).

Os corpos de prova foram secos em estufa a $110^{\circ} \mathrm{C}$ por $24 \mathrm{~h} \mathrm{e}$, em seguida, foram submetidos a queima em forno elétrico de laboratório (Maitec, FE50PM) nas temperaturas de $800,900,1000,1100$ e $1200{ }^{\circ} \mathrm{C}$, em atmosfera oxidante, com taxa de aquecimento de $5{ }^{\circ} \mathrm{C} / \mathrm{min}$. O tempo de permanência na temperatura máxima foi de 60 e $180 \mathrm{~min}$. Foram analisadas as propriedades de retração linear de queima, absorção de água e resistência mecânica à flexão em três pontos (velocidade do braço de aplicação da carga de $0,5 \mathrm{~mm} / \mathrm{min}$ ). As fases formadas após queima foram analisadas por difração de raios X (Shimadzu, XRD 6000). As análises estatísticas dos resultados foram efetuadas através da comparação das médias utilizando o teste $t$ de Student, com a análise das variâncias usando o teste $\mathrm{F}$ de Snedecor.

\section{RESULTADOS E DISCUSSÃO}

Os resultados de composição química das argilas analisadas encontram-se na Tabela I. Observou-se predominância de $\mathrm{SiO}_{2}$ e $\mathrm{Al}_{2} \mathrm{O}_{3}$, o que é característico dos aluminossilicatos presentes nas argilas. Com relação aos teores de alumina $\left(\mathrm{Al}_{2} \mathrm{O}_{3}\right)$, que é um indicativo da proporção de mineral argiloso e do feldspato presente nas argilas, verificou-se que as argilas $\mathrm{A}$ e $\mathrm{B}$ apresentaram valores bem próximos. A argila $\mathrm{C}$ apresentou a menor razão $\mathrm{Al}_{2} \mathrm{O}_{3} / \mathrm{SiO}_{2}$, sugerindo que esta apresentou uma maior quantidade de sílica livre. Verificou-se também que a amostra $\mathrm{C}$ apresentou

Tabela I - Composição química (\% em massa) das argilas.

[Table I - Chemical composition (wt\%) of the clays.]

\begin{tabular}{ccccccccccc}
\hline Amostra & $\mathrm{SiO}_{2}$ & $\mathrm{Al}_{2} \mathrm{O}_{3}$ & $\mathrm{Fe}_{2} \mathrm{O}_{3}$ & $\mathrm{~K}_{2} \mathrm{O}$ & $\mathrm{MgO}$ & $\mathrm{CaO}$ & $\mathrm{TiO}_{2}$ & Outros & $\mathrm{PF}^{\mathrm{a}}$ & Total \\
\hline Argila A & 46,1 & 32,1 & 5,4 & 1,9 & 1,1 & 0,2 & 0,7 & 0,8 & 11,7 & 100 \\
Argila B & 47,1 & 33,0 & 5,1 & 1,5 & 0,9 & 0,1 & 0,6 & 0,3 & 11,4 & 100 \\
Argila C & 44,2 & 24,3 & 8,1 & 3,6 & 2,4 & 1,3 & 1,0 & 2,1 & 13,0 & 100 \\
\hline aPF: perda ao fogo. & & & & & & & & & &
\end{tabular}




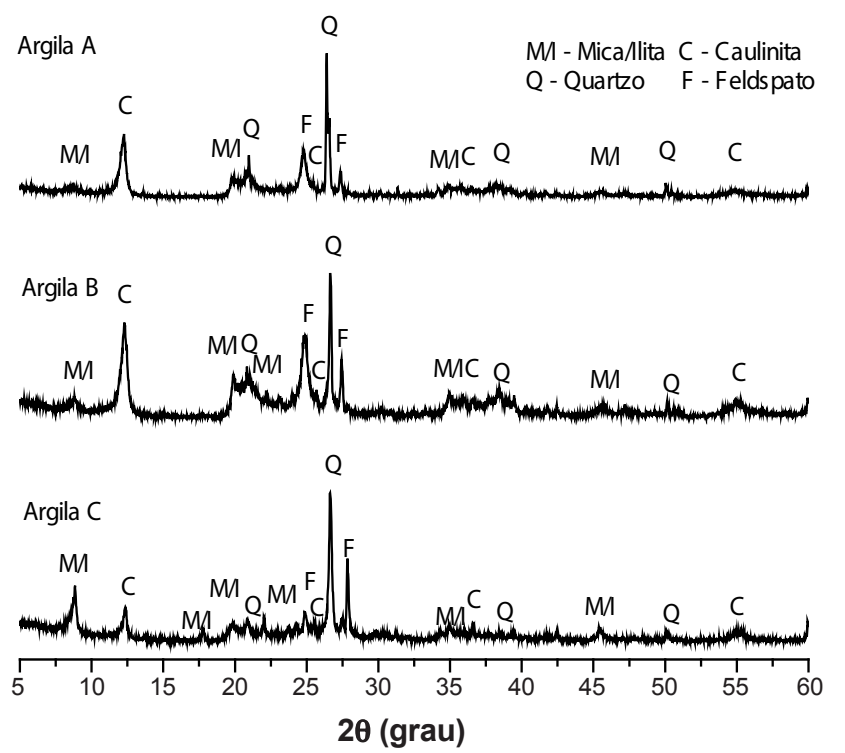

Figura 1: Difratogramas de raios $\mathrm{X}$ das argilas.

[Figure 1: X-ray diffraction patterns of the clays.]

os maiores teores de $\mathrm{Fe}_{2} \mathrm{O}_{3}$ e de óxidos fundentes. Quanto aos óxidos alcalinos e alcalino terrosos $\left(\mathrm{K}_{2} \mathrm{O}, \mathrm{MgO}\right.$ e $\left.\mathrm{CaO}\right)$, importantes para formação da fase vítrea durante a queima, pôde-se observar que as argilas A e B apresentaram teores superiores a $2,5 \%$, enquanto a amostra $\mathrm{C}$ apresentou teor significativamente elevado, superior a $7 \%$. Destaca-se que não foi detectada a presença de $\mathrm{Na}_{2} \mathrm{O}$ em nenhuma das argilas. Os valores de perda ao fogo, que é característica da presença de água livre, adsorvida e de constituição das matérias-primas, como também da oxidação da matéria orgânica, foram similares aos encontrados em trabalhos da literatura [4-6], que também analisaram características de

Tabela II - Dados da distribuição granulométrica de tamanho de partículas e limites de Atterberg.

[Table II - Particle size distribution data and Atterberg limits.]

\begin{tabular}{|c|c|c|c|c|c|c|}
\hline \multirow{2}{*}{ Argila } & \multicolumn{3}{|c|}{$\begin{array}{c}\text { Fração acumulada } \\
(\%)\end{array}$} & \multicolumn{3}{|c|}{$\begin{array}{c}\text { Limites de Atterberg } \\
(\%)\end{array}$} \\
\hline & $\begin{array}{l}<2 \\
\mu \mathrm{m}\end{array}$ & $\begin{array}{c}2 \text { a } 20 \\
\mu \mathrm{m}\end{array}$ & $\begin{array}{l}>20 \\
\mu \mathrm{m}\end{array}$ & $\mathrm{LL}^{\mathrm{a}}$ & $\mathrm{LP}^{\mathrm{b}}$ & $\mathrm{IP}^{\mathrm{c}}$ \\
\hline A & 16,05 & 53,24 & 30,71 & 36,65 & 18,86 & 17,79 \\
\hline B & 8,49 & 42,89 & 48,62 & 37,80 & 20,82 & 16,98 \\
\hline $\mathrm{C}$ & 11,49 & 46,61 & 41,90 & 31,20 & 15,08 & 16,12 \\
\hline
\end{tabular}

${ }^{a} L L$ : limite de liquidez; ${ }^{b} L P$ : limite de plasticidade; ${ }^{c} I P:$ índice de plasticidade. argilas de queima vermelha utilizadas na indústria cerâmica.

Os difratogramas das argilas analisadas, Fig. 1, apresentaram picos característicos das seguintes fases cristalinas: caulinita (JCPDS 14-0164), mica/ilita (JCPDS 83-1808), quartzo (JCPDS 46-1045) e feldspato (JCPDS 89-8575). A amostra B apresentou picos de caulinita de maior intensidade e a amostra $\mathrm{C}$ picos de mica/ilita e de feldspato mais intensos. Comparando os difratogramas com os apresentados em trabalhos da literatura $[14,15]$, verificou-se que as massas apresentaram fases cristalinas semelhantes às reportadas para argilas usadas em cerâmica estrutural.

Na Tabela II constam os dados obtidos a partir da distribuição granulométrica do tamanho de partículas e os limites de Atterberg das argilas analisadas. Observou-se que a argila A apresentou composição granulométrica de acordo com o diagrama de Winkler [16] para a produção de tijolos maciços. No entanto, as argilas B e C apresentaram fração argila, $<2 \mu \mathrm{m}$, abaixo do mínimo indicado para massas visando a fabricação de produtos da cerâmica vermelha. Por outro lado, estudos [17, 18] abordando massas utilizadas para fabricação de produtos de cerâmica tradicional observaram que entre as formulações utilizadas com êxito para produção de corpos de cerâmica vermelha havia massas que se ajustavam e outras que não se adequavam às regiões delimitadas pelo diagrama de Winkler para este fim. A distribuição granulométrica de uma massa cerâmica interfere significativamente sobre a plasticidade, preenchimento do molde e empacotamento do sistema e tem íntima relação com a densificação, as necessidades de secagem e as características de processamento do material. No entanto, apenas a definição da granulometria pode não expressar a adequabilidade do material a determinado uso, sendo necessário levar em consideração as condições de processamento e de sua interação com as características das matérias-primas, tal como evidenciado nos artigos mencionados [17, 18], para se ter uma definição da adequabilidade ou não de determinada matéria-prima argilosa para o uso pretendido.

Com relação aos limites de Atterberg, verificou-se que as argilas apresentaram valores de IP (índice de plasticidade) dentro do intervalo observado para cerâmica vermelha e foram classificadas como altamente plásticas (IP $>15$ ). Observou-se ainda que a argila $\mathrm{A}$ apresentou maior plasticidade, o que está de acordo com o observado na distribuição granulométrica, já que esta apresentou a maior fração abaixo de $2 \mu \mathrm{m}$. Mesmo com menor fração abaixo de $2 \mu \mathrm{m}$, a argila B apresentou índice de plasticidade adequado para produção de cerâmicas estruturais. Analisando

Tabela III - Densidade a seco $\left(\mathrm{g} / \mathrm{cm}^{3}\right)$ dos corpos de prova das argilas A, B e C. [Table III - Dry density $\left(\mathrm{g} / \mathrm{cm}^{3}\right)$ of specimens of clays A, B and C.]

\begin{tabular}{cccccc}
\hline \multicolumn{2}{c}{ Argila A } & \multicolumn{2}{c}{ Argila B } & \multicolumn{2}{c}{ Argila C } \\
Prensagem & Extrusão & Prensagem & Extrusão & Prensagem & Extrusão \\
\hline $1,60 \pm 0,02$ & $1,68 \pm 0,01$ & $1,63 \pm 0,01$ & $1,71 \pm 0,02$ & $1,81 \pm 0,01$ & $1,84 \pm 0,01$ \\
\hline
\end{tabular}


trabalhos realizados [4, 19], observou-se que as argilas analisadas apresentaram parâmetros de trabalhabilidade que as possibilitam ser conformadas tanto por prensagem quanto por extrusão.

Na Tabela III encontram-se os resultados da densidade a seco dos corpos de prova das argilas A, B e C. De acordo com os dados apresentados, observa-se que, quando conformadas por extrusão, as amostras apresentaram maior densidade a seco comparada com a conformação por prensagem (teste $t$ a um nível de significância de 5\%), obtendo-se valor de $\mathrm{p}$ inferior a $0,01 \mathrm{em}$ todas as argilas. Verificou-se um aumento de $5 \%$ na densificação dos corpos confeccionados com as argilas A e B e um aumento em torno de $2 \%$ quando do uso da argila $C$, após processo de extrusão. Este comportamento foi, provavelmente, relacionado com a plasticidade da massa associada à maior pressão de compactação inerente ao processo de extrusão, que proporcionou melhor deslizamento entre as partículas, favorecendo um maior empacotamento das partículas. Ademais, a extrusão proporciona um empacotamento mais homogêneo ao longo do corpo o que, aliado à maior densificação, possibilita obter corpos após queima com melhor desempenho mecânico, tendo importância fundamental no comportamento final dos materiais [11].

A Tabela IV apresenta os resultados da retração linear de queima dos corpos de prova produzidos com as argilas A, B e C. De acordo com os dados apresentados, observase que os maiores valores encontrados para retração linear de queima foram para os corpos de prova submetidos à queima a $1200{ }^{\circ} \mathrm{C}$, comprovando a relação entre a retração e a temperatura de queima. A retração linear de queima apresentou oscilações com o aumento da temperatura de queima, sendo, em geral, maior (teste $t, p<0,05$ ) para os ciclos em que o tempo de permanência na temperatura máxima foi de $180 \mathrm{~min}$. Conforme os resultados obtidos, verificou-se que as argilas A e B apresentaram valores de retração linear semelhantes nas cinco temperaturas de queima, apesar da diferença nas características granulométricas das duas argilas (Tabela II). É interessante destacar que as amostras A e B apresentaram maiores retrações comparativamente à amostra $\mathrm{C}$ até $1100{ }^{\circ} \mathrm{C}$, enquanto a argila $\mathrm{C}$ apresentou valores de retração superiores ou similares após queima a $1200^{\circ} \mathrm{C}$ (teste $\mathrm{t}, \mathrm{p}<0,05)$. Segundo $[20,21]$, geralmente argilas com maior quantidade de quartzo mostram menor contração linear, fato que pode explicar o comportamento observado, pois a argila $\mathrm{C}$ apresentou menor relação $\mathrm{Al}_{2} \mathrm{O}_{3} / \mathrm{SiO}_{2}$, sugerindo que esta apresentou uma maior quantidade de sílica livre. No entanto, para a queima a $1200^{\circ} \mathrm{C}$, esta apresentou uma maior retração quando conformada por prensagem, destacando-se a maior quantidade de fundentes presentes nesta massa, os quais fundem a temperatura acima de $1150{ }^{\circ} \mathrm{C}$ produzindo a fase líquida responsável pela densificação dos corpos de prova. Verificou-se também que os resultados de retração linear foram maiores para as argilas conformadas por extrusão. Fato que pode ser explicado devido ao maior grau de homogeneidade das massas argilosas e maior aproximação das partículas proporcionada pela extrusão.

$\mathrm{Na}$ Tabela $\mathrm{V}$ encontram-se os resultados da absorção de água dos corpos de prova das argilas A, B e C. De acordo com os dados apresentados, verifica-se que as argilas A e $\mathrm{B}$, quando submetidas às temperaturas de queima de 800 , 900 e $1000{ }^{\circ} \mathrm{C}$, apresentaram valores de absorção de água da ordem de 21 a $25 \%$, sendo superiores a $20 \%$, valor máximo admissível para fabricação de telhas, e próximos a $25 \%$, valor máximo admissível para fabricação de blocos [22], enquanto que os corpos de prova da argila $\mathrm{C}$ apresentaram valores de absorção de água abaixo de $20 \%$ em todas as temperaturas de queima. Os valores elevados de absorção de água podem estar relacionados com o empacotamento após compactação e ao menor teor de fundentes dessas argilas. Verificou-se que as argilas apresentaram um decréscimo significativo nos valores de absorção de água à medida que a temperatura de queima aumentou para 1100 e $1200{ }^{\circ} \mathrm{C}$. Acima de $1000{ }^{\circ} \mathrm{C}$ ocorreu a formação de fase líquida, que contribuiu para o processo de sinterização, diminuindo a porosidade devido ao preenchimento dos poros, ocorrendo desse modo densificação. Pôde-se observar que o aumento do tempo de queima provocou diminuição nos valores de

Tabela IV - Retração linear de queima (\%) dos corpos de prova das argilas A, B e C. [Table IV - Linear firing shrinkage of specimens of clays A, B and C.]

\begin{tabular}{cccccccc}
\hline $\begin{array}{c}\text { Temperatura } \\
\left({ }^{\circ} \mathrm{C}\right)\end{array}$ & $\begin{array}{c}\text { Tempo } \\
(\mathrm{min})\end{array}$ & \multicolumn{2}{c}{ Argila A } & \multicolumn{2}{c}{ Argila B } & \multicolumn{2}{c}{ Argila C } \\
\hline \multirow{2}{*}{800} & 60 & $0,93 \pm 0,05$ & $1,14 \pm 0,12$ & $0,98 \pm 0,15$ & $0,57 \pm 0,11$ & $0,16 \pm 0,01$ & $0,12 \pm 0,12$ \\
& 180 & $0,87 \pm 0,04$ & $1,04 \pm 0,13$ & $0,93 \pm 0,04$ & $0,85 \pm 0,13$ & $0,20 \pm 0,08$ & $0,16 \pm 0,18$ \\
\multirow{2}{*}{900} & 60 & $1,54 \pm 0,34$ & $1,69 \pm 0,22$ & $1,68 \pm 0,22$ & $1,68 \pm 0,17$ & $0,24 \pm 0,01$ & $0,18 \pm 0,13$ \\
& 180 & $1,62 \pm 0,01$ & $1,91 \pm 0,05$ & $1,79 \pm 0,10$ & $1,93 \pm 0,17$ & $0,28 \pm 0,08$ & $0,34 \pm 0,11$ \\
& 60 & $1,88 \pm 0,07$ & $2,27 \pm 0,11$ & $1,97 \pm 0,24$ & $2,03 \pm 0,18$ & $0,51 \pm 0,07$ & $1,79 \pm 0,12$ \\
1000 & 180 & $1,98 \pm 0,02$ & $2,43 \pm 0,10$ & $2,12 \pm 0,07$ & $2,28 \pm 0,12$ & $0,61 \pm 0,05$ & $1,72 \pm 0,13$ \\
& 60 & $4,92 \pm 0,07$ & $7,29 \pm 0,12$ & $4,57 \pm 0,16$ & $7,38 \pm 0,07$ & $3,11 \pm 0,04$ & $4,46 \pm 0,15$ \\
1100 & 180 & $5,88 \pm 0,07$ & $8,37 \pm 0,24$ & $5,97 \pm 0,03$ & $8,92 \pm 0,12$ & $3,50 \pm 0,21$ & $4,33 \pm 0,15$ \\
& 60 & $6,82 \pm 0,15$ & $9,23 \pm 0,19$ & $6,49 \pm 0,01$ & $7,24 \pm 0,13$ & $8,33 \pm 0,08$ & $8,26 \pm 0,17$ \\
1200 & 180 & $7,90 \pm 0,17$ & $9,84 \pm 0,14$ & $7,57 \pm 0,04$ & $10,13 \pm 0,14$ & $8,88 \pm 0,21$ & $8,86 \pm 0,19$ \\
\hline
\end{tabular}


Tabela $V$ - Absorção de água (\%) dos corpos de prova das argilas $A, B$ e $C$.

[Table V-Water absorption (\%) of the test specimens of the clays $A, B$ and $C$.]

\begin{tabular}{|c|c|c|c|c|c|c|c|}
\hline \multirow{2}{*}{ Temperatura $\left({ }^{\circ} \mathrm{C}\right)$} & \multirow{2}{*}{$\begin{array}{c}\text { Tempo } \\
\text { (min) }\end{array}$} & \multicolumn{2}{|c|}{ Argila A } & \multicolumn{2}{|c|}{ Argila B } & \multicolumn{2}{|c|}{ Argila C } \\
\hline & & Prensagem & Extrusão & Prensagem & Extrusão & Prensagem & Extrusão \\
\hline \multirow{2}{*}{800} & 60 & $25,31 \pm 0,23$ & $22,02 \pm 0,11$ & $24,41 \pm 0,30$ & $21,27 \pm 0,24$ & $16,35 \pm 0,14$ & $15,07 \pm 0,23$ \\
\hline & 180 & $22,66 \pm 0,25$ & $21,87 \pm 0,13$ & $21,19 \pm 0,55$ & $21,04 \pm 0,28$ & $17,04 \pm 0,05$ & $15,06 \pm 0,16$ \\
\hline \multirow{2}{*}{900} & 60 & $25,16 \pm 0,06$ & $21,31 \pm 0,13$ & $24,64 \pm 0,21$ & $21,18 \pm 0,18$ & $16,33 \pm 0,20$ & $14,24 \pm 0,24$ \\
\hline & 180 & $22,80 \pm 0,20$ & $21,47 \pm 0,09$ & $22,51 \pm 0,31$ & $21,04 \pm 0,17$ & $17,01 \pm 0,26$ & $14,08 \pm 0,15$ \\
\hline \multirow{2}{*}{1000} & 60 & $24,44 \pm 1,18$ & $20,08 \pm 0,55$ & $23,83 \pm 0,12$ & $20,35 \pm 0,24$ & $15,22 \pm 0,04$ & $11,18 \pm 0,11$ \\
\hline & 180 & $21,91 \pm 0,21$ & $20,65 \pm 0,34$ & $21,36 \pm 0,85$ & $20,85 \pm 0,36$ & $15,58 \pm 0,14$ & $10,78 \pm 0,23$ \\
\hline \multirow{2}{*}{1100} & 60 & $16,22 \pm 0,04$ & $8,87 \pm 0,24$ & $16,26 \pm 0,32$ & $8,98 \pm 0,16$ & $9,93 \pm 0,04$ & $7,01 \pm 0,34$ \\
\hline & 180 & $13,85 \pm 0,01$ & $7,42 \pm 0,11$ & $14,56 \pm 0,23$ & $6,92 \pm 0,15$ & $10,54 \pm 0,43$ & $6,97 \pm 0,25$ \\
\hline \multirow{2}{*}{1200} & 60 & $13,01 \pm 0,14$ & $6,15 \pm 0,23$ & $12,44 \pm 0,10$ & $5,27 \pm 0,14$ & $3,05 \pm 0,35$ & $2,06 \pm 0,06$ \\
\hline & 180 & $10,70 \pm 0,24$ & $4,16 \pm 0,12$ & $11,12 \pm 0,03$ & $3,99 \pm 0,23$ & $1,10 \pm 0,13$ & $1,20 \pm 0,10$ \\
\hline
\end{tabular}

absorção de água das amostras A e B (teste t, $\mathrm{p}<0,05$ ), em todas as temperaturas de queima quando processadas por prensagem, enquanto que quando processadas por extrusão observou-se esse comportamento apenas nas temperaturas de queima de 1100 e $1200{ }^{\circ} \mathrm{C}$ (teste $\mathrm{t}, \mathrm{p}<0,05$ ). Por outro lado, verificou-se que ocorreu um aumento na absorção de água (teste $\mathrm{t}, \mathrm{p}<0,05$ ) nas amostras prensadas após queima a 800 e $900{ }^{\circ} \mathrm{C}$, e não alteração na temperatura de queima de $1000{ }^{\circ} \mathrm{C}$, quando do aumento do tempo de queima. Esse comportamento pode estar associado à presença de elevada quantidade de mica nessa amostra e a desidroxilação e destruição da sua estrutura nessas temperaturas de queima. No que tange aos métodos de processamento, observouse que a extrusão possibilitou, de forma geral, diminuição da absorção de água em todas as temperaturas de queima e em todas as argilas (com base no teste $t$ a um nível de significância de $5 \%, \mathrm{p}<0,05)$. Nesse sentido, os corpos de prova da argila $\mathrm{C}$ foram os que apresentaram os menores valores de absorção de água, o que pode estar relacionado à maior quantidade de fundentes dessa argila e ao maior empacotamento propiciado pela maior quantidade de quartzo nesse material.

Na Tabela VI encontram-se os resultados da resistência mecânica à flexão, pelo método de três pontos, dos corpos de prova das argilas A, B e C. De acordo com os dados apresentados (teste $t, p<0,05$ ), observa-se que, de forma geral, à medida que foi elevada a temperatura de queima ocorreu aumento dos valores de resistência à flexão para as amostras. Para as temperaturas de queima de 1100 e $1200{ }^{\circ} \mathrm{C}$, quando do uso do processo de conformação por prensagem, estas apresentaram valores dentro dos recomendados para blocos furados $(\geq 5,5 \mathrm{MPa})$ e para telhas ( $\geq 6,5 \mathrm{MPa}$ ), sendo que os corpos de prova das argilas A e B não apresentaram valores acima do mínimo de referência de

Tabela VI - Resistência à flexão (MPa) dos corpos de prova das argilas A, B e C.

[Table VI-Flexural strength (MPa) of the specimens of the clays A, B and C.]

\begin{tabular}{|c|c|c|c|c|c|c|c|}
\hline \multirow{2}{*}{$\begin{array}{c}\text { Temperatura } \\
\left({ }^{\circ} \mathrm{C}\right)\end{array}$} & \multirow{2}{*}{$\begin{array}{l}\text { Tempo } \\
\text { (min) }\end{array}$} & \multicolumn{2}{|c|}{ Argila A } & \multicolumn{2}{|c|}{ Argila B } & \multicolumn{2}{|c|}{ Argila C } \\
\hline & & Prensagem & Extrusão & Prensagem & Extrusão & Prensagem & Extrusão \\
\hline \multirow{2}{*}{800} & 60 & $1,43 \pm 0,25$ & $6,03 \pm 0,16$ & $1,47 \pm 0,10$ & $5,98 \pm 0,13$ & $1,60 \pm 0,12$ & $7,75 \pm 0,26$ \\
\hline & 180 & $2,55 \pm 0,06$ & $7,78 \pm 0,75$ & $2,39 \pm 0,10$ & $7,23 \pm 0,46$ & $1,51 \pm 0,11$ & $7,97 \pm 0,38$ \\
\hline \multirow{2}{*}{900} & 60 & $1,89 \pm 0,14$ & $9,39 \pm 0,48$ & $1,73 \pm 0,08$ & $10,23 \pm 0,37$ & $2,05 \pm 0,14$ & $10,39 \pm 0,36$ \\
\hline & 180 & $3,32 \pm 0,07$ & $10,68 \pm 0,41$ & $2,28 \pm 0,15$ & $9,82 \pm 0,52$ & $1,96 \pm 0,05$ & $10,32 \pm 0,37$ \\
\hline \multirow{2}{*}{1000} & 60 & $1,94 \pm 0,13$ & $11,56 \pm 0,55$ & $2,33 \pm 0,21$ & $11,23 \pm 0,60$ & $2,65 \pm 0,16$ & $10,21 \pm 0,47$ \\
\hline & 180 & $3,50 \pm 0,11$ & $12,91 \pm 0,69$ & $3,28 \pm 0,21$ & $11,55 \pm 0,59$ & $2,32 \pm 0,17$ & $10,49 \pm 0,63$ \\
\hline \multirow{2}{*}{1100} & 60 & $4,93 \pm 0,17$ & $22,79 \pm 0,53$ & $4,81 \pm 0,36$ & $20,58 \pm 0,60$ & $7,01 \pm 0,28$ & $24,46 \pm 0,80$ \\
\hline & 180 & $9,55 \pm 0,28$ & $25,20 \pm 0,65$ & $6,64 \pm 0,41$ & $24,40 \pm 0,59$ & $6,30 \pm 0,22$ & $23,69 \pm 0,69$ \\
\hline \multirow{2}{*}{1200} & 60 & $9,39 \pm 0,32$ & $27,53 \pm 0,64$ & $9,15 \pm 0,40$ & $24,36 \pm 0,62$ & $17,40 \pm 0,36$ & $28,26 \pm 0,42$ \\
\hline & 180 & $15,98 \pm 0,41$ & $31,77 \pm 0,52$ & $11,98 \pm 0,39$ & $28,78 \pm 0,65$ & $20,56 \pm 0,50$ & $35,26 \pm 0,63$ \\
\hline
\end{tabular}


5,5 MPa para a temperatura de queima de $1100{ }^{\circ} \mathrm{C}$ quando permaneceram durante 60 min nesta temperatura. Já quando o processo de conformação usado foi a extrusão, os valores se apresentaram acima do mínimo de referência a partir da temperatura de queima de $800{ }^{\circ} \mathrm{C}$, independentemente da argila utilizada no processo. Os resultados obtidos vão ao encontro de trabalhos apresentados na literatura [23, 24], utilizando massas para cerâmica vermelha. Nesses estudos observou-se que as peças conformadas com as massas com maiores quantidades de óxidos fundentes apresentaram maiores densificação e resistência mecânica.
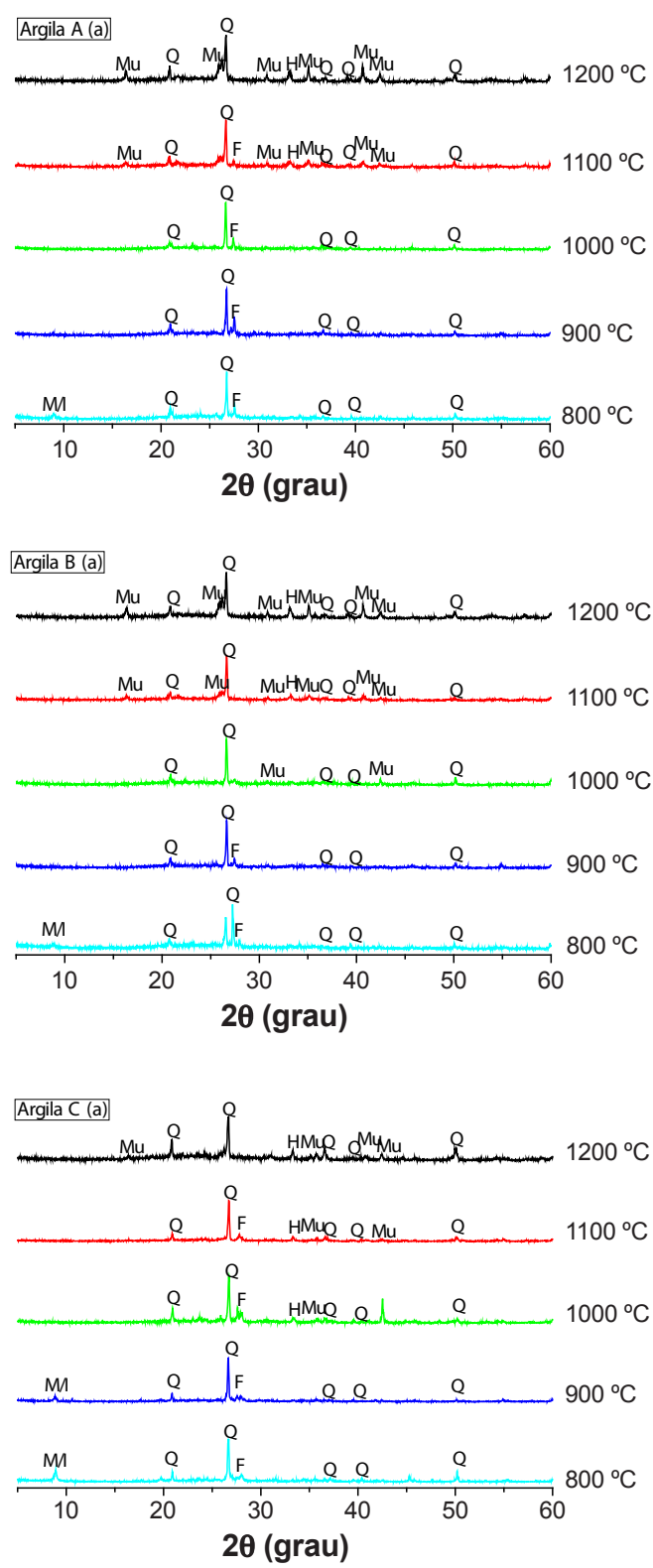

Comparando os tempos de permanência na temperatura máxima durante a queima das amostras, observou-se que a resistência mecânica à flexão foi maior (teste $t, p<0,05$ ) nos corpos da argila A para um tempo de permanência de 180 min, independentemente do tipo de método de conformação. Já nos corpos produzidos com a argila B verificou-se uma elevação na resistência mecânica (teste $t, p<0,05$ ) apenas nos corpos produzidos por prensagem e nos extrudados queimados nas temperaturas de 1100 e $1200^{\circ} \mathrm{C}$. Nos corpos obtidos com a amostra $\mathrm{C}$ verificou-se que o aumento do tempo de queima não provocou alteração na resistência
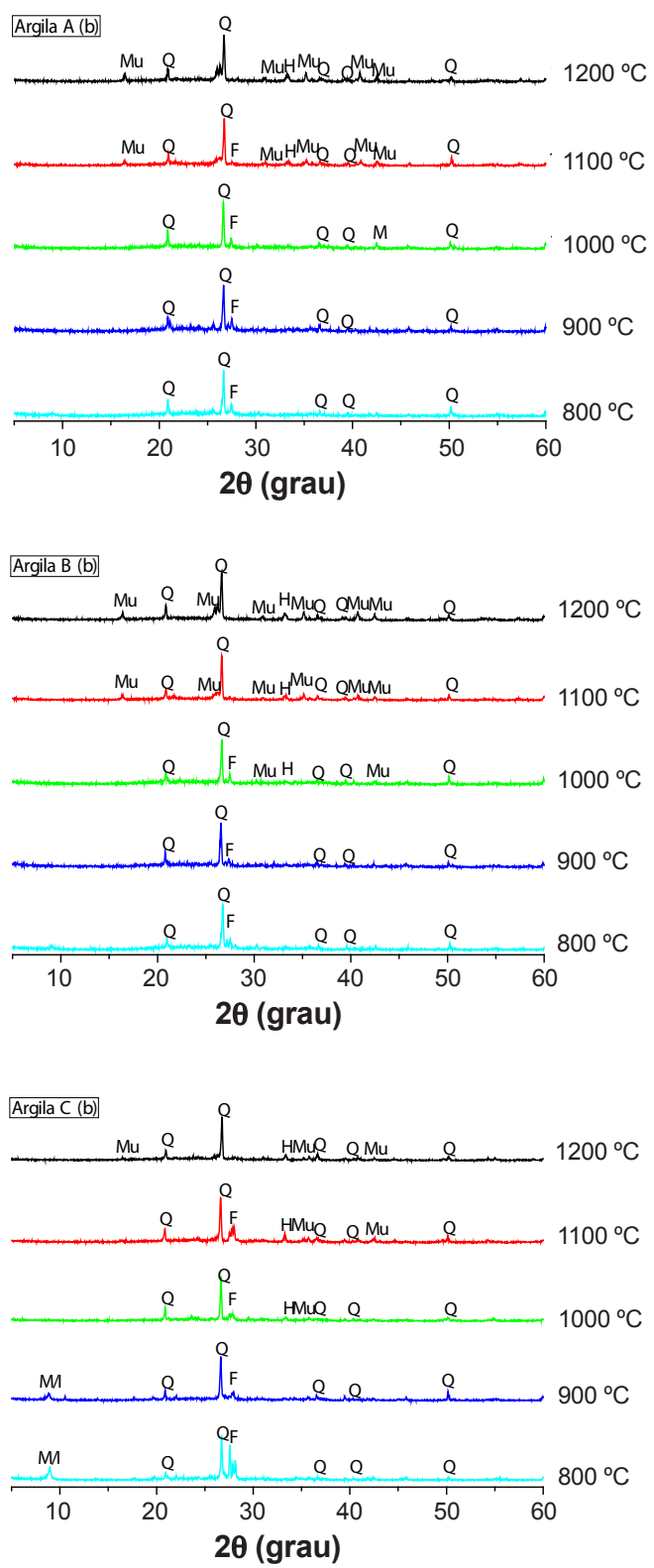

M/I-mica/ilita; Q-quartzo; F-feldspato; H-hematita; Mu-mulita

Figura 2: Difratogramas de raios X dos produtos das argilas A, B e C, conformados por prensagem (a) e por extrusão (b), sinterizados a 800, $900,1000,1100$ e $1200{ }^{\circ} \mathrm{C}$ com taxa de aquecimento de $5{ }^{\circ} \mathrm{C} /$ min e tempo de permanência de 180 min na temperatura máxima.

[Figure 2: X-ray diffraction patterns of the products of the clays A, B and C shaped by pressing (a) and extrusion (b), sintered at 800, 900, 1000,1100 and $1200^{\circ} \mathrm{C}$, with heating rate of $5^{\circ} \mathrm{C} / \mathrm{min}$ and holding time of $180 \mathrm{~min}$.] 
(800 e $\left.900{ }^{\circ} \mathrm{C}\right)$ ou causou diminuição da resistência à flexão dos corpos $\left(1000\right.$ e $1100^{\circ} \mathrm{C}$, teste $\left.t, p<0,05\right)$, quando da queima em temperaturas inferiores a $1200{ }^{\circ} \mathrm{C}$. Esse comportamento vem ao encontro do observado para a absorção de água e, como mencionado anteriormente, pode estar relacionado ao teor de mica presente na amostra C. Os corpos extrudados da amostra $\mathrm{C}$, tal como os da amostra $\mathrm{B}$, apresentaram melhora na resistência com o aumento do tempo de permanência apenas nas temperaturas de queima de 1100 e $1200^{\circ} \mathrm{C}$. Provavelmente, o maior tempo de permanência na temperatura máxima favoreceu o melhor equilíbrio entre as reações químicas e físicas, ou seja, o desenvolvimento de uma microestrutura com menor quantidade de defeitos, conforme observado em [2] em estudo realizado com massas para confecção de produtos da cerâmica vermelha. Outros pesquisadores $[16,25]$ também analisaram a influência dos parâmetros de queima sobre as propriedades mecânicas de materiais cerâmicos e observaram que as reações físico-químicas que ocorrem durante esse tratamento térmico foram determinantes para produzir peças de cerâmica vermelha com propriedades mecânicas melhoradas, devendo-se fundamentalmente ao fechamento da porosidade aberta no interior das peças de cerâmica vermelha. Analisando conjuntamente as propriedades físico-mecânicas das amostras estudadas e conformadas pelos processos de prensagem e extrusão, ficou evidenciado a influência da interação entre as partículas e do processo de conformação; pode-se observar que o processo de extrusão proporcionou maior grau de compactação das massas argilosas, contribuindo para se obter maior resistência mecânica nos corpos de prova.

Os difratogramas das amostras queimadas estãoapresentados na Fig. 2. Constatou-se a presença dos picos característicos das seguintes fases cristalinas: mica/ilita (JCPDS 83-1808), quartzo (JCPDS 46-1045), feldspato (JCPDS 89-8575) e hematita (JCPDS 33-0664), além de picos característicos de mulita (JCPDS 18-1225). Verificaram-se picos de mulita de baixa intensidade e picos de quartzo com maior intensidade, mesmo após queima a $1200{ }^{\circ} \mathrm{C}$, o que foi relacionado à presença de quartzo grosseiro nas amostras, dimensões superiores a 45 $\mu \mathrm{m}$. A formação de novas fases passa por uma reorganização estrutural quando a decomposição térmica ocorre, modificando as propriedades físicas dos corpos cerâmicos. Observou-se que as três amostras apresentaram basicamente os mesmos picos característicos das fases cristalinas supracitadas quando conformadas tanto por prensagem quanto por extrusão. Em trabalhos realizados [2, 14] também foram estudadas as transformações térmicas de massas utilizadas para a fabricação de produtos da cerâmica vermelha, e estes observaram que durante o aquecimento inicialmente a caulinita foi transformada em metacaulinita, e com a continuação do aquecimento, entre 800 e $1000{ }^{\circ} \mathrm{C}$, surgiu uma fase amorfa rica em $\mathrm{SiO}_{2}$ e a fase mulita, as quais foram fundamentais para as propriedades tecnológicas das amostras.

\section{CONCLUSÕES}

Este trabalho teve como finalidade avaliar a influência das características iniciais das massas e de diferentes parâmetros de processamento sobre as propriedades tecnológicas dos corpos cerâmicos. Com base nos resultados experimentais pôde-se concluir que o processo de extrusão se sobrepôs quando comparado com o processo de prensagem, uma vez que a extrusão proporcionou resistências significativamente superiores. Além disso, verificou-se que o aumento do tempo de permanência na temperatura máxima de queima proporcionou o melhor balanceamento entre as reações químicas e físicas, impactando na fabricação de peças com propriedades mecânicas melhoradas. O estudo mostrou que a interação entre as características granulométricas, mineralógicas e químicas dependem do tipo de processo de conformação utilizado e do ciclo de queima estabelecido, acarretando consequentemente alterações sobre as propriedades tecnológicas do produto final.

\section{AGRADECIMENTOS}

Os autores agradecem a CAPES e ao CNPq (309646/20138) pelo apoio financeiro e aos colegas do Laboratório de Tecnologia de Materiais, LTM, da Universidade Federal de Campina Grande, pela ajuda no trabalho experimental, em especial a Rennaly Mota e José Vieira Neto.

\section{REFERÊNCIAS}

[1] F.L. Formiga, J.C.S. Andrade, P.A.S. Araújo, D.A. Macedo, A.E. Martinelli, R.M. Nascimento, C.A. Paskocimas, Cerâm. Ind. 18 (2013) 30.

[2] E. Escalera, R. Tegman, M.L. Antti, M. Odén, Appl. Clay Sci. 101 (2014) 100.

[3] D. Lahcen, E.E. Hichama, S. Latifa, A. Abderrahmane, B. Jamal, W. Mohamed, E. Meriam, F. Nathalie, Appl. Clay Sci. 102 (2014) 139.

[4] A. Bennour, S. Mahmoudi, E. Srasra, N. Hatira, S. Boussen, M. Ouaja, F. Zargouni, Appl. Clay Sci. 118 (2015) 212.

[5] S. Mahmoudi, A. Bennour, A. Meguebli, E. Srasra, F. Zargouni, Appl. Clay Sci. 127-128 (2016) 78.

[6] P.D. Ndjigui, J.A. Mbey, A.N. Nzeukou, J. Build. Eng. 5 (2016) 151.

[7] V. Lee, T. Yeh, Mater. Sci. Eng. A 485 (2008) 5.

[8] R.S. Macedo, R.R. Menezes, G.A. Neves, H.C. Ferreira, Cerâmica 54, 332 (2008) 411.

[9] J.L.A. Albero, Cerâm. Ind. 5 (2000) 23.

[10] N.S. Muller, G. Vekinis, V. Kilikoglou, J. Archaeol. Sci.: Reports 7 (2016) 519.

[11] C.S.R. Freitas, I.A.F.S. Pereira, M.L.G. Pereira, T.C.R. Simões, A.A.L. Ferreira, Cerâm. Ind. 14 (2009) 15.

[12] J.L.A. Amorós, Cerâm. Ind. 5 (2000) 23.

[13] M. Cargnin, S.M.A.G.U. de Souza, A.A.U. de Souza, A.J. Noni, Cerâmica 57, 344 (2011) 461.

[14] A. Bennour, S. Mahmoudi, E. Srasra, S. Boussen, N. Hatira, Appl. Clay Sci. 115 (2015) 30.

[15] M. Dondi, M. Raimindo, C.H. Zanelli, Appl. Clay Sci. 96 (2014) 91.

[16] G.P Souza, R. Sanchez, J.N.F. Holanda, Cerâmica 48, 
306 (2002) 102.

[17] M.M. Jordan, F. Pardo, T. Sanfeliu, S. Meseguer, Appl. Clay Sci. 101 (2014) 619.

[18] D. Eliche-Quesada, C. Martínez-García, M.L. MartínezCartas, M.T. Cotes-Palomino, L. Pérez-Villarejo, N. CruzPérez, F. A. Corpas-Iglesias, Appl. Clay Sci. 52 (2011) 270.

[19] F.H. Zaied, R. Abidi, N. Slim-Shimi, A.K. Somarin, Appl. Clay Sci. 112 (2015) 1.

[20] A. Barba, V. Beltán, C. Feliu, J. Garcia, F. Gines, E. Sánchez, V. Sanz, Materias primas para la fabricación de soportes de baldosas cerámicas, Instituto de Tecnología Cerámica, AICE, Espanha (1997) 138.
[21] A.N. Nzeukou, N. Fagel, A. Njoya, V.B. Kamgang, R.E. Medjo, U.C. Melo, Appl. Clay Sci. 83-84 (2013) 238.

[22] P.S. Santos, Ciência e tecnologia de argilas, $2^{\mathrm{a}} \mathrm{Ed}$., Edgard Blücher, Vol. I e II, S. Paulo (1992).

[23] M.M. Jordan, M.A. Montero, S. Meseguer, T. Sanfeliu, Appl. Clay Sci. 42 (2008) 266.

[24] S. Mustafi, M. Ahsan, A.H. Dewan, S. Ahmed, N. Khatun, N. Absar, J. Sci. Res. 24 (2011) 169.

[25] E.T. Stepkowska, S.A. Jefferis, Appl. Clay Sci. 6 (1992) 319.

(Rec. 19/07/2016, Rev. 08/10/2016, 21/12/2016, Ac. 21/12/2016) 